\title{
BANACH BUNDLES OF CONTINUOUS FUNCTIONS AND AN INTEGRAL REPRESENTATION THEOREM
}

\author{
BY
}

ANTHONY KAREL SEDA

\begin{abstract}
A construction is given of a Banach bundle $p: A \rightarrow X$ whose fibres are spaces of continuous functions which vanish at infinity. A Riesz type integral representation theorem is established which describes all functionals on $A$.
\end{abstract}

1. Introduction. Mappings $p: A \rightarrow X$, loosely called bundles, whose fibres are endowed with Banach space structures have been encountered in analysis for some considerable time. For example, direct integrals of Hilbert spaces and fields of operators arise naturally in considering unitary representations of topological groups. In this context, $X$ is a measure space and $A$ is the set-theoretic union of Hilbert spaces $A_{x}, x \in X$; one is primarily concerned here with forming $L^{2}$ spaces of cross sections. A similar situation arises in describing representations of a topological group induced from representations of its subgroups. In this case, $X$ is a coset space equipped with a measure (-class) and again one forms a type of $L^{2}$ space of sections. However, this process involves complications from a measure-theoretic point of view and these tend to obscure the construction.

A conceptually clearer approach to this inducing process has been provided by Fell $[4,5]$. In Fell's setting, $A$ and $X$ are topological spaces, $p$ is continuous and open and $p: A \rightarrow X$ is required to satisfy certain natural axioms making it a Banach bundle. Essentially the same object is used in representing a $C^{*}$-algebra a by sections of a bundle (or sheaf) of $C^{*}$-algebras over the primitive ideal space $X$ of a. This point of view has been extensively developed over the last decade by Dupré, Fell, Hofmann, Mulvey et al.

Our immediate goal here is to give the construction of a Banach bundle (Theorem 1) which parallels the classical Banach space $C_{0}(X)$, of continuous functions which vanish at infinity on $X$, and to describe the functionals defined on it (Theorem 2). Our description amounts to a version of the Riesz representation theorem in the bundle context, and was inspired by the application which we give in $\$ 4$ to prove continuity of the convolution product defined there. This product arises in the study of induced representations of groups and has been treated in some detail in [6]. It is worth noting, too, that Theorem 2 is to some extent complementary to the version of the Hahn-Banach theorem given by Burden in [3] for normed linear spaces in the category of sheaves on $X$.

Received by the editors February 19, 1981.

1980 Mathematics Subject Classification. Primary 22D30, 46H15; Secondary 28C05.

(c) 1982 American Mathematical Society $0002-9947 / 81 / 0000-1037 / \$ 02.50$ 
2. Banach bundles of continuous functions. Given a locally compact space $Y$, we shall employ the following notation: $k(Y)$ denotes the space of complex valued continuous functions in $Y$ with compact support; for compact $K \subseteq Y, k(Y, K)$ denotes the subspace of $k(Y)$ consisting of those functions whose support is inside $K$ equipped with the uniform norm; $C_{0}(Y)$ denotes the uniform-norm completion of $k(Y)$, that is, the space of continuous functions on $Y$ which vanish at infinity.

Suppose $q: Y \rightarrow X$ is continuous, open and surjective, where $X$ and $Y$ are locally compact Hausdorff. For each $x \in X$ let $Y_{x}$ denote the fibre $q^{-1}(x)$ of $q$ and let $A_{x}=C_{0}\left(Y_{x}\right)$ equipped with the uniform norm, \|\|$_{x}$. Let $A=\cup_{x \in X} A_{x}$ and let $p$ : $A \rightarrow X$ be the projection defined by $p^{-1}(x)=A_{x}$. Given $\phi \in k(Y)$, there is an induced cross sectional function $\tilde{\phi}: X \rightarrow A$ defined by $\tilde{\phi}(x)=\left.\phi\right|_{Y_{x}}$. In fact, $\tilde{\phi}(x) \in k\left(Y_{x}\right), \tilde{\phi}_{1}=\tilde{\phi}_{2}$ if, and only if, $\phi_{1}=\phi_{2}$ and the set $\Gamma=\{\tilde{\phi} ; \phi \in k(Y)\}$ forms a complex vector space.

Theorem 1. (i) For each $x \in X$, the set $\{\tilde{\phi}(x) ; \tilde{\phi} \in \Gamma\}$ is dense in $A_{x}$.

(ii) For each $\tilde{\phi} \in \Gamma$, the numerical function $\|\tilde{\phi}(x)\|_{x}$ is continuous on $X$.

(iii) $p: A \rightarrow X$ is a Banach bundle and each $\tilde{\phi}$ is continuous and is, hence, a section of $p$.

Proof. (i) Suppose $f \in k\left(Y_{x}\right)$. Then the support of $f$ is compact in $Y$ and so $f$ can be extended by the Tietze extension theorem to a function $\phi \in k(Y)$. Hence $\tilde{\phi}(x)=f$ and (i) follows.

(ii) Let $K$ be a compact neighbourhood of the support of $\phi$ in $Y$, let $x \in X$ be fixed and suppose $\varepsilon>0$. For any $\alpha \in K_{x}=K \cap Y_{x}$ there is, by continuity of $\phi$, a neighbourhood $U(\alpha)$ of $\alpha$ in $Y$, with $U(\alpha) \subseteq \operatorname{int}(K)$, such that $|\phi(\alpha)-\phi(\beta)|<\varepsilon / 2$ for all $\beta \in U(\alpha)$. Moreover, the $U(\alpha)$ can be taken to be maximal with these properties. By compactness of $K_{x}$ and openness of $q$, finitely many of these neighbourhoods $U\left(\alpha_{j}\right), j=1,2, \ldots, n$, cover $K_{x}$ and $\cap_{j=1}^{n} q\left(U\left(\alpha_{j}\right)\right)$ is a neighbourhood of $x$ in $X$.

Claim. There is a neighbourhood $O$ of $x$ in $X, O \subseteq \cap_{j=1}^{n} q\left(U\left(\alpha_{j}\right)\right)$, with the property that $\operatorname{supp}(\tilde{\phi}(y)) \subseteq \cup_{j=1}^{n} U\left(\alpha_{j}\right)$ whenever $y \in O$.

If no such $O$ exists, there is a net $y_{\lambda} \rightarrow x$ in $X$ and a net $\alpha_{\lambda}$ in $K$ such that:

(1) $q\left(\alpha_{\lambda}\right)=y_{\lambda}$ for each $\lambda$.

(2) $\left|\phi\left(\alpha_{\lambda}\right)-\phi\left(\alpha_{j}\right)\right| \geqslant \varepsilon / 2$ for all $\lambda, j=1,2, \ldots, n$.

Since $K$ is compact, there is a subnet $\alpha_{\lambda \mu}$ of $\alpha_{\lambda}$ converging in $K$ to $\beta$, say, in which case $q\left(\alpha_{\lambda \mu}\right)$ converges to $q(\beta)$ in $X$ and it follows that $q(\beta)=x$, since $q\left(\alpha_{\lambda \mu}\right)$ is a subnet of $y_{\lambda}$. But then $\left|\phi(\beta)-\phi\left(\alpha_{j}\right)\right| \geqslant \varepsilon / 2$ for each $j$, which is a contradiction since $\beta \in K_{x}$. This proves the claim.

Let $O$ be a neighbourhood of $x$ satisfying the claim and let $y \in O$. Let $\beta^{\prime}$ and $\alpha^{\prime}$ be elements of $\operatorname{supp}(\phi)$ which achieve $\|\tilde{\phi}(y)\|$ and $\|\tilde{\phi}(x)\|$, respectively. Then for any $j$ such that $\beta^{\prime} \in U\left(\alpha_{j}\right)$ we have $\left|\phi\left(\beta^{\prime}\right)-\phi(\alpha)\right|<\varepsilon$ for all $\alpha \in U\left(\alpha_{j}\right)$, from which we obtain $\left|\phi\left(\beta^{\prime}\right)\right|<\left|\phi\left(\alpha^{\prime}\right)\right|+\varepsilon$. Similarly, $\left|\phi\left(\alpha^{\prime}\right)\right|<\left|\phi\left(\beta^{\prime}\right)\right|+\varepsilon$ and so $|\|\tilde{\phi}(y)\|-\|\tilde{\phi}(x)\||<\varepsilon$ for all $y \in O$, as required.

(iii) This follows from [4, Proposition 1.6]. 
3. Integral representation of functionals on $A$. In this section we shall establish our main result, giving an integral representation of functionals defined on the Banach bundle $A$ constructed in $\S 2$.

Let $M_{+}(Y)$ denote the cone of all positive measures on $Y$ endowed with the vague topology of Bourbaki [2], in which a net $\mu_{\lambda}$ of measures converges to $\mu$ if, and only if, $\mu_{\lambda}(f) \rightarrow \mu(f)$ for each $f \in k(Y)$. We shall identify any Baire measure [1] on $Y$ with the positive functional (that is, the measure in the sense of Bourbaki) it determines and vice versa using the classical Riesz representation theorem.

Now suppose that for each $x \in X$ we have Baire measure $\mu_{x}$ on $Y$ with $\operatorname{supp}\left(\mu_{x}\right) \subseteq Y_{x}$ and that $\mathrm{M}: X \rightarrow M_{+}(Y), \mathrm{M}(x)=\mu_{x}$, is vaguely continuous. This latter requirement is equivalent to the continuity of the scalar function $\mu_{x}(f)$ on $X$ for each $f \in k(Y)$.

Proposition 1. Let $H=\left\{\mu_{x} ; x \in X\right\}$. Then $H$ is a vaguely bounded subset of $M_{+}(Y)$.

Proof. Let $K \subseteq Y$ be compact, let $C$ be a compact neighbourhood of $K$ and use Urysohn's lemma to obtain a nonnegative $\phi \in k(Y)$ such that $\phi=1$ on $K$ and $\operatorname{supp}(\phi) \subseteq C$. Let $M_{K}=\sup \left\{\mu_{x}(\phi) ; x \in X\right\}$. Then for any $\mu_{x} \in H$ and $f \in k(Y, K)$, we have

$$
\left|\mu_{x}(f)\right| \leqslant \mu_{x}(|f|) \leqslant \mu_{x}(\|f\| \phi) \leqslant M_{K}\|f\|
$$

and the result follows from [2, $\S 1$, No. 9, Proposition 15].

Definition. Let $p: E \rightarrow X$ be any Banach bundle over $X$ and let $\mathfrak{F}$ (either $\mathbf{C}$ or $R$ ) denote the scalar field of each of the Banach spaces $E_{x}$. Then a map $T: E \rightarrow \mathfrak{F}$ is called a functional if $T_{x}=\left.T\right|_{E_{x}}$ is a linear functional on $E_{x}$ for each $x \in X$. In the case $E=A$, as in $\S 2$, a functional $T$ will be called positive if $T_{x}$ is positive for each $x \in X$.

It will be convenient to record here one fact concerning the topology of the total space $E$ of a Banach bundle $p: E \rightarrow X$, where $X$ is locally compact Hausdorff. Given any point $e \in E$, there exists a section $\sigma$ of $p$ passing through $e$ in the sense that $\sigma(p(e))=e$. This basic result, due to Douady and dal Soglio-Hérault, can be found in the appendix of [5]. It follows that sets of the type $W=W(\sigma, V, \varepsilon)=$ $\{s \in E ; p(s) \in V,\|s-\sigma(p(s))\|<\varepsilon\}$ form a neighbourhood base at $e$, where $\varepsilon>0$ and $V$ is a neighbourhood of $p(e)$.

Proposition 2. Let $p: E \rightarrow X$ be a Banach bundle and $T: E \rightarrow \mathbf{C}$ a continuous functional. Then $\left\|T_{x}\right\|$ is locally bounded on $X$.

Proof. Since $T$ is continuous at the origin $O(x)$ of $E_{x}$ and the zero section $O$ is continuous, there is an $\varepsilon>0$ and a neighbourhood $V$ of $x$ such that $T(W(O, V, \varepsilon)) \subseteq D$, where $D$ is the unit disc. By linearity of the $T_{y}$ the set $\{s \in E ; p(s) \in V,\|s\|<2\}=2 W / \varepsilon$ is mapped by $T$ inside $2 D / \varepsilon$. Hence, $\left\|T_{y}\right\| \leqslant 2 / \varepsilon$ for all $y \in V$ as required. 
Returning to $p: A \rightarrow X$ as in $\S 2$ we prove

THEOREM 2. Suppose $T: A \rightarrow \mathbf{C}$ is a continuous positive functional on $A$. Then there exists a unique family $H=\left\{\mu_{x} ; x \in X\right\}$ of Baire measures $\mu_{x}$ on $Y$ such that:

(1) $\mu_{x}$ is bounded and $\operatorname{supp}\left(\mu_{x}\right) \subseteq Y_{x}$.

(2) $\left\|\mu_{x}\right\|=\left\|T_{x}\right\|$.

(3) The function $\left\|\mu_{x}\right\|$ is locally bounded on $X$.

(4) $\mathrm{M}: X \rightarrow M_{+}(Y)$, where $\mathrm{M}(x)=\mu_{x}$, is vaguely continuous.

(5) $T(f)=\mu_{p(f)}(f)$ for all $f \in A$.

Conversely, if $H$ is a family of Baire measures on $Y$ satisfying (1), (3) and (4), then the expression (5) determines a continuous positive functional on A satisfying (2).

Proof. Since $T_{x}$ is a bounded linear functional on $C_{0}\left(Y_{x}\right)$ there exists, by the classical theory, a unique bounded Baire measure $\mu_{x}$ on $Y_{x}$ such that $T_{x}(f)=\mu_{x}(f)$ for all $f \in A_{x}$. We may regard $\mu_{x}$ as a Baire measure on $Y$ with support contained in $Y_{x}$ in the usual way, and so (1) and (5) follow and (2) also by the classical theory. Since (3) now follows from Proposition 2, it remains to establish (4). Let $\phi \in k(Y)$; then $\tilde{\phi}: X \rightarrow A$ is continuous by Theorem 1 . Hence the composite $T \tilde{\phi}$ is continuous and $(T \tilde{\phi})(x)=\mu_{x}(\phi)$. Thus, $\mathrm{M}$ is vaguely continuous.

Conversely, suppose $\left\{\mu_{x} ; x \in X\right\}$ satisfies (1), (3) and (4). Then the expression $T_{x}(f)=\mu_{x}(f), f \in A_{x}$, determines a positive functional on $A$ which satisfies (2). For continuity of $T$, let $f \in A_{x}$ be arbitrary and suppose $\varepsilon>0$. By (3) there is a positive number $B$ and a neighbourhood $V$ of $x$ in $X$ such that $\left\|T_{y}\right\| \leqslant B$ for all $y \in V$. By Theorem 1 there is a $\tilde{\phi} \in \Gamma$ such that $\|f-\tilde{\phi}(x)\|<\varepsilon /(4(B+1))$. Next observe that the function $T \tilde{\phi}: X \rightarrow \mathbf{C}$ is continuous by (4), and so, by shrinking $V$ if necessary, we can suppose that $|(T \tilde{\phi})(y)-(T \tilde{\phi})(x)|<\varepsilon / 2$ for all $y \in V$. Finally, let $W=W(\tilde{\phi}, V, \varepsilon / 4 B)$; then $W$ is a neighbourhood of $f$ in $A$ and if $s \in W$, we have $\|s-\tilde{\phi}(p(s))\|<\varepsilon / 4 B$. Hence, whenever $s \in W$,

$$
\begin{aligned}
|T s-T f| & \leqslant|T s-T(\tilde{\phi}(p(s)))|+|(T \tilde{\phi}) p(s)-(T \tilde{\phi}) x|+|(T \tilde{\phi}) x-T f| \\
& \leqslant\left\|T_{p(s)}\right\|\|s-\tilde{\phi}(p(s))\|+\varepsilon / 2+\left\|T_{x}\right\|\|\tilde{\phi}(x)-f\| \\
& <B \varepsilon / 4 B+\varepsilon / 2+B \varepsilon / 4(B+1)<\varepsilon .
\end{aligned}
$$

Thus, $T$ is continuous and the proof is complete.

4. The convolution algebra $k(G)$. Let $G$ be a locally compact Hausdorff topological groupoid over $X$ and let $\left\{\mu_{x} ; x \in X\right\}$ be a Haar measure on $G$ (see [6] and [7]). We shall make the following

Assumption. M: $X \rightarrow M_{+}(G)$, where $\mathrm{M}(x)=\mu_{x}$, is vaguely continuous.

Under this assumption it follows that the initial and final maps $\pi, \pi^{\prime}: G \rightarrow X$ are open mappings. This fact is due to J. Westman (see [6, Proposition 2.4]).

Now suppose $f, g \in k(G)$ and define their convolution product $f * g$ by

$$
(f * g)(\alpha)=\int_{G} f(\beta) g\left(\beta^{-1} \alpha\right) d \mu_{\pi^{\prime}(\alpha)}(\beta)
$$


for each $\alpha \in G$. This section will be devoted to applying Theorem 2 to prove

THEOREM 3. The multiplication * endows $k(G)$ with the structure of an associative algebra over $\mathbf{C}$.

4.1 Remarks concerning Theorem 3. This theorem clearly generalises the group case, and the first form of the theorem to do this was given in [9]. However, Westman assumes that $G$ is locally trivial and his Haar measure is essentially different from ours. The next version was given by us, see [8] and the references there, again assuming $G$ locally trivial, and also in the case of the groupoid associated with a transformation group. The final and present form of the theorem was first published in [6] and obtained independently by us. However, the proof we give below is quite different from Renault's and brings out the bundle-theoretic nature of the problem. Renault shows, in fact, that $(k(G), *)$ is a topological algebra in the inductive limit topology on $k(G)$ determined by the $k(G, K), K \subseteq G$ compact. One may find in [6] and its references a treatment of the role played by $k(G)$ in the Murray-von Neumann group measure space construction, and also in the work of Effros and Hahn on transformation group $C^{*}$ algebras.

4.2 Proof of Theorem 3. The only nontrivial steps in the proof concern * . If $\operatorname{supp}(f)=K$ and $\operatorname{supp}(g)=L$, it is easy to see that $\operatorname{supp}(f * g)$ is contained in the compact set $K \cdot L$, which is the image under the composition law in $G$ of $K \times L$. Moreover, once we have shown $f * g$ is continuous, associativity of $*$ follows by the invariance of the measures $\mu_{x}$ and an application of Fubini's theorem. We content ourselves with proving that $f * g$ is continuous.

Let $C$ be a compact neighbourhood of the support, $K$, of $f$ and let $\Phi$ be a continuous real valued nonnegative function on $G$ such that $\Phi=1$ on $K$ and $\operatorname{supp}(\Phi) \subseteq C$. Let $\mu_{x}^{\prime}$ be the scalar product $\Phi \mu_{x}$ for each $x \in X$. Then $\mu_{x}^{\prime}$ is bounded, $\operatorname{supp}\left(\mu_{x}^{\prime}\right) \subseteq Y_{x},\left\|\mu_{x}^{\prime}\right\|$ is locally bounded by Proposition 1 and $\mu_{x}^{\prime}$ is vaguely continuous in $x$. Let $p: A \rightarrow X$ be the bundle constructed as in Theorem 1 relative to the map $q=\pi^{\prime}: G \rightarrow X$, and let $T: A \rightarrow \mathbf{C}$ be the functional provided by Theorem 2 relative to the measures $\mu_{x}^{\prime}$.

For each $\alpha \in G$ define $F_{\alpha}$ by $F_{\alpha}(\beta)=f(\beta) g\left(\beta^{-1} \alpha\right)$ for all $\beta$ such that $\pi^{\prime}(\beta)=\pi^{\prime}(\alpha)$. Then $F_{\alpha} \in A, \operatorname{supp}\left(F_{\alpha}\right) \subseteq K$ and it is clear that $(f * g)(\alpha)=T\left(F_{\alpha}\right)$. So it suffices to show that the function $\alpha \mapsto F_{\alpha}$ mapping $G$ into $A$ is continuous, since $T$ is continuous.

Suppose $\alpha_{0} \in G$ and $\pi^{\prime}\left(\alpha_{0}\right)=y_{0}$. Let $\tilde{\phi}$ be a section of $p$ passing through $F_{\alpha_{0}}$ with $\operatorname{supp}(\phi) \subseteq C$ and let $W=W(\tilde{\phi}, V, \varepsilon)$ be a basic neighbourhood of $F_{\alpha_{0}}$. Now

$$
\left\|F_{\alpha}-\tilde{\phi}\left(\pi^{\prime}(\alpha)\right)\right\|=\sup _{\beta}\left|f(\beta) g\left(\beta^{-1} \alpha\right)-\phi(\beta)\right|
$$

and this vanishes at $\alpha=\alpha_{0}$. By precisely the same argument as used to prove Theorem 1(ii), there is a neighbourhood $O$ of $\alpha_{0}$ in $G$ such that if $\alpha \in O$, then $\left|f(\beta) g\left(\beta^{-1} \alpha\right)-\phi(\beta)\right|<\varepsilon / 2$ for all $\beta \in O$ such that $\beta^{-1} \alpha$ is defined, in which case $\left\|F_{\alpha}-\tilde{\phi}\left(\pi^{\prime}(\alpha)\right)\right\|<\varepsilon$ and so $F_{\alpha}$ is continuous in $\alpha$ as required. 


\section{REFERENCES}

1. S. K. Berberian, Measure and integration, Macmillan, New York, 1965.

2. N. Bourbaki, Intégration. III: Eléments de mathématique, Hermann, Paris, 1959.

3. C. W. Burden, The Hahn-Banach theorem in a category of sheaves, J. Pure Appl. Algebra 17 (1980), $25-34$.

4. J. M. G. Fell, An extension of Mackey's method to Banach*-algebraic bundles, Mem. Amer. Math. Soc. No. 90 (1969).

5. __ Induced representations and Banach *-algebraic bundles, Lecture Notes in Math., vol. 582, Springer-Verlag, Berlin and New York, 1977.

6. J. Renault, A groupoid approach to $C^{*}$-algebras, Lecture Notes in Math., vol. 793, Springer-Verlag, Berlin and New York, 1980.

7. A. K. Seda, Haar measures for groupoids, Proc. Roy. Irish Acad. Sect. A 76 (1976), 25-36.

8. Quelques résultats dans la catégorie des groupoides d'opérateurs, C. R. Acad. Sci. Paris Sér. A-B 288 (1979), 21-24.

9. J. Westman, Harmonic analysis on groupoids, Pacific J. Math. 27 (1968), 621-632.

Department of Mathematics, University College, Cork, Ireland 